Address correspondence to: Alan N. Houghton, Memorial Sloan-Kettering Cancer Center, 1275 York Avenue, New York, New York 10021, USA. Phone: (212) 639-7595; Fax: (212) 794-4352; E-mail: a-houghton@ski.mskcc.org.

1. Burnet, F.M. 1961. Immunological recognition of self. Science. 133:307-311.

2. Houghton, A.N., Gold, J.S., and Blachere, N.E. 2001. Immunity against cancer: lessons learned from melanoma. Curr. Opin. Immunol. 13:134-140.

3. Hahn, W.C., and Weinberg, R.A. 2002. Rules for making human tumor cells. N. Engl. J. Med. 347:1593-1603.

4. Land, H., Parada, L.F., and Weinberg, R.A. 1983. Tumorigenic conversion of primary embryo fibroblasts requires at least two cooperating oncogenes. Nature. 304:596-602.

5. Prehn, R.T., and Main, J.M. 1957. Immunity to methylcholanthrene-induced sarcomas. J. Natl. Cancer Inst. 18:769-778.

6. Lurquin, C., et al. 1989. Structure of the gene of tum- transplantation antigen P91A: the mutated exon encodes a peptide recognized with Ld by cytolytic T cells. Cell. 58:293-303.

7. Dubey, P., et al. 1997. The immunodominant antigen of an ultraviolet-induced regressor tumor is generated by a somatic point mutation in the DEAD box helicase p68. J. Exp. Med. 185:695-705.

8. Hewitt, H.B., Blake, E.R., and Walder, A.S. 1976. A critique of the evidence for active host defense against cancer, based on personal studies of 27 murine tumours of spontaneous origin. Br. J. Cancer. 33:241-259.

9. Naftzger, C., et al. 1996. Immune response to a differentiation antigen induced by altered antigen: a study of tumor rejection and autoimmunity. Proc. Natl. Acad. Sci. U. S. A. 93:14809-14814.

10. Weber, L.W., et al. 1998. Tumor immunity and autoimmunity induced by immunization with homologous DNA. J. Clin. Invest. 102:1258-1264.

11. Overwijk, W.W., et al. 1998. gp100/pmel 17 is a murine tumor rejection antigen: induction of "self"reactive, tumoricidal $\mathrm{T}$ cells using high-affinity, altered peptide ligand. J. Exp. Med. 188:277-286.

12. Bowne, W.B., et al. 1999. Coupling and uncoupling of tumor immunity and autoimmunity. J. Exp. Med. 190:1717-1722.

13. Gold, J.S., et al. 2003. A single heteroclitic epitope determines cancer immunity after xenogeneic DNA immunization against a tumor differentia- tion antigen. J. Immunol. 170:5188-5194.

14. Van Pel, A., Vessiere, F., and Boon, T. 1983. Protection against two spontaneous mouse leukemias conferred by immunogenic variants obtained by mutagenesis. J. Exp. Med. 157:1992-2001.

15. Boon, T., and Van Pel, A. 1978. Teratocarcinoma cell variants rejected by syngeneic mice: protection of mice immunized with these variants against other variants and against the original malignant cell line. Proc. Natl. Acad. Sci. U. S. A. 75:1519-1523.

16. Yu, Z., et al. 2004. Poor immunogenicity of a self/ tumor antigen derives from peptide-MHC-I instability and is independent of tolerance. J. Clin. Invest. 114:551-559. doi:10.1172/JCI200421695.

17. Houghton, A.N. 1994. Cancer antigens. Immune recognition of self and altered self. J. Exp. Med. 180:1-4.

18. Dyall, R., et al. 1998. Heteroclitic immunization induces tumor immunity. J. Exp. Med. 188:1553-1561.

19. Houghton, A.N., et al. 1985. Mouse monoclonal IgG3 antibody detecting GD3 ganglioside: a phase I trial in patients with malignant melanoma. Proc. Natl. Acad. Sci. U. S. A. 82:1242-1246.

20. Rasttter, W., Molina, A., White, C.A. 2004. Rituximab: expanding role in therapy for lymphomas and autoimmune diseases. Annu. Rev. Med. 55:477-503.

\title{
The T cell cometh: interplay between adaptive immunity and cytokine networks in rheumatoid arthritis
}

\author{
Gary S. Firestein
}

Division of Rheumatology, Allergy, and Immunology and Clinical Investigation Institute, University of California San Diego School of Medicine, San Diego, California, USA.

The etiology of autoimmunity in humans remains poorly defined, and animal models provide a unique opportunity to study potential autoimmune mechanisms. A novel model of autoimmune inflammatory arthritis results from a point mutation in the $\zeta$-associated-protein of $70 \mathrm{kDa}$ (ZAP-70), which causes abnormal thymic $T$ cell selection and survival of autoreactive clones (see the related article beginning on page 582). Although the resulting clinical and pathologic abnormalities are clearly $T$ cell-dependent, macrophage and fibroblast cytokines such as IL-1 and TNF- $\alpha$ are required for full expression of the disease. The studies of Hata et al. raise the intriguing possibility that traditional proinflammatory cytokine networks represent common effector mechanisms in inflammatory joint diseases such as rheumatoid arthritis. Hence, effective therapeutic interventions can target either unique etiologic pathways related to adaptive immune responses or shared terminal mechanisms.

Nonstandard abbreviations used: $\zeta$-associated protein of $70 \mathrm{kDa}$ (ZAP-70); collagen-induced arthritis (CIA); cyclic citrullinated peptide (CCP); Grb2-associated binder 2 (Gab2); linker for activation of T cells (LAT); lymphocyte protein tyrosine kinase (Lck); rheumatoid arthritis (RA); SH2-domain-containing leukocyte protein of $76 \mathrm{kDa}$ (SLP-76); Src homology 2 (SH2); vaccinia virus VH1-related (VHR).

Conflict of interest: The author has declared that no conflict of interest exists.

Citation for this article: J. Clin. Invest. 114:471-474 (2004). doi:10.1172/JCI200422651.
Like Sisyphus, who was doomed to forever push a boulder up a mountain only to see it roll down again, investigators attempting to develop models of rheumatoid arthritis (RA) have endured a frustrating existence. Many rodent strains engineered over the last few decades have been touted as "RA in a mouse (or rat)," and only later have the limitations and differences from human disease been recognized. Howev- er, with each model, we learn something new about the pathogenesis of synovial inflammation that can move the field one step closer to an effective therapy.

In this issue of the JCI, Hata and colleagues (1) have extended their insightful work on a novel inflammatory arthritis model resembling RA caused by a mutation in the gene encoding $\zeta$-associated protein of $70 \mathrm{kDa}$ (ZAP-70). In normal immune responses, CD4-associated lymphocyte protein tyrosine kinase (Lck) phosphorylates the $\xi$-chain of the $\mathrm{T}$ cell receptor-CD3 complex after antigen engagement (2). ZAP-70 is then recruited to the complex and is also phosphorylated by Lck (Figure 1). Dephosphorylation of inhibitory sites on ZAP-70 by low-molecular weight phosphotyrosine phosphatase further enhances ZAP-70 function. Several downstream targets subsequently serve as substrates for ZAP-70 kinase activity, including linker for activation of $\mathrm{T}$ cells (LAT), Grb2-associated binder 2 (Gab2), Src homology 2 (SH2) domain-containing leukocyte protein of 


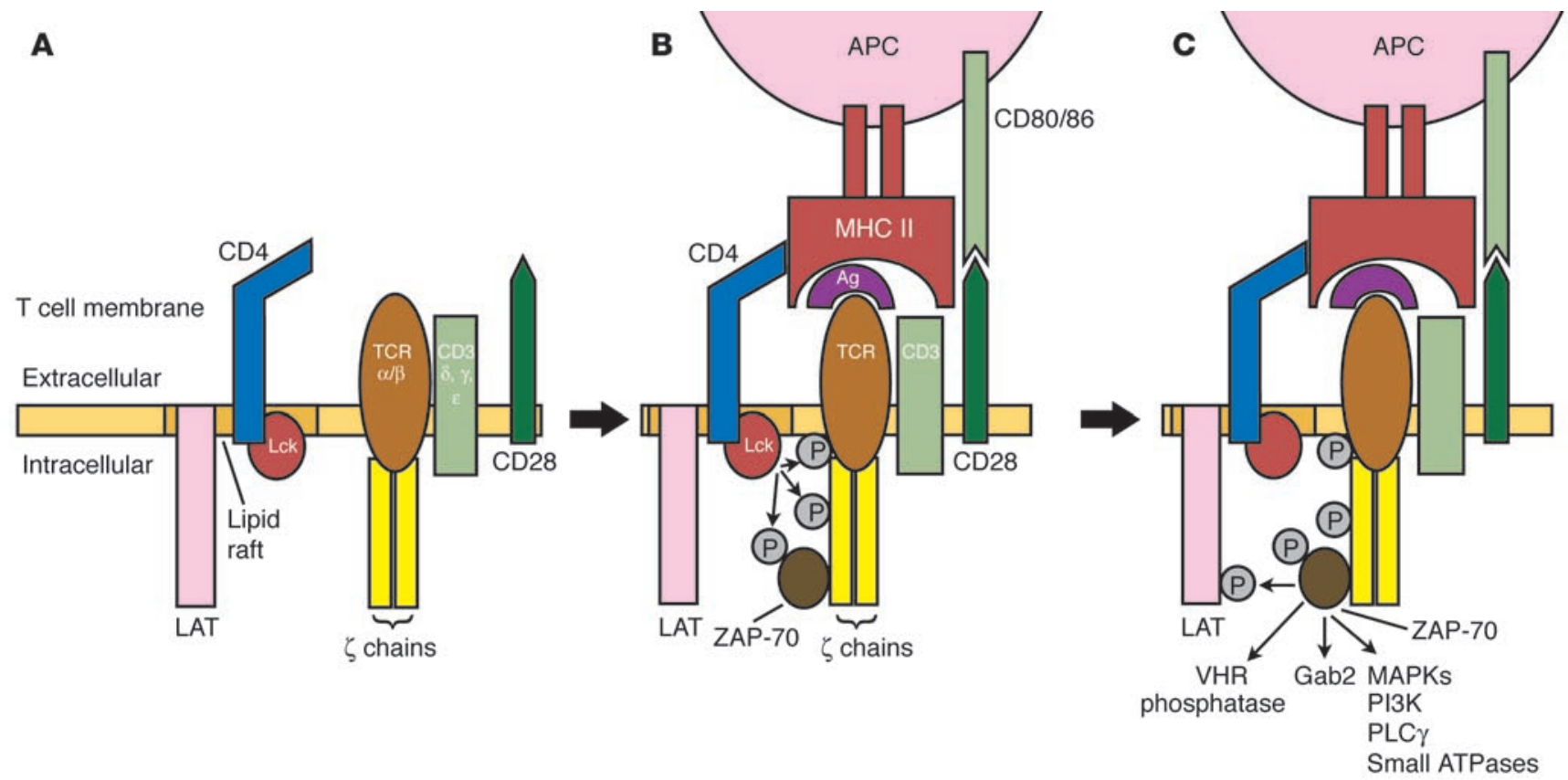

Figure 1

Sequence of events implicated in naive T cell activation. The T cell receptor (TCR) complex, including the CD3 complex and $\zeta$-chains, are expressed by resting naive T cells adjacent to lipid rafts that contain LAT, CD4, and kinases like Lck (A). Ligation of the TCR by antigen and MHC class II protein recruits CD4 and costimulatory molecules like CD28, which engage MHC and CD80/86, respectively. Activated Lck and other kinases then phosphorylate $(\mathrm{P})$ the $\xi$-chain, which, in turn, recruits ZAP-70 (B). ZAP-70 is also phosphorylated by Lck and other kinases in this process and can, in turn, phosphorylate numerous downstream substrates, including MAPKs and many other signaling pathways (C). PLC $\gamma$, phospholipase C $\gamma$.

$76 \mathrm{kDa}(\mathrm{SLP}-76)$, vaccinia virus VH1-related (VHR) phosphatase, and many other signaling molecules.

The specific mutation observed in the SKG mouse, which results in an amino acid change from tryptophan to cysteine at codon 163 , leads to abnormal thymic $\mathrm{T}$ cell selection and inappropriate survival of autoreactive clones (3). Spontaneous symmetric inflammatory arthritis develops in the mice after about 2 months, with erosions, synovial hyperplasia, and rheumatoid factor production. Other autoantibodies that recognize joint antigens, such as type II collagen, are also detected in the blood of these mice. Anticyclic citrullinated peptide (anti-CCP) antibody levels, which are relatively specific for RA, have not been reported. CD4 ${ }^{+}$ T cells, thymocytes, and bone marrow cells passively transfer the disease. SKG serum containing abundant autoantibodies is not pathogenic when injected into normal mice (S. Sakaguchi, personal communication), which suggests an essential difference from the $\mathrm{K} / \mathrm{BxN}$ mouse model of RA, which begins as a $T$ cell-dependent response to glucose-6-phosphoisomerase but later is solely dependent on autoantibodies (4).
The clinical and histologic features of the SKG model resemble RA although there are important differences. For instance, neutrophils rarely infiltrate rheumatoid synovium but are abundant in the SKG joint lining. Skin inflammation as observed in the mice is not a feature of RA, and the prevalence of pulmonary fibrosis is very low in humans compared with that in the animal model. The mice also develop distal interphalangeal joint disease and dactylitis, which are both uncommon in RA. ZAP-70 function in RA is, as in SKG mice, suppressed. However - and this is a critical distinction - this is the result of synovial inflammation and altered redox balance in active synovitis, and ZAP-70 function returns to normal after the disease is suppressed (5). Hence, ZAP-70 abnormalities are generally caused by RA rather than vice versa.

These caveats do not detract from the seminal observation that altered $\mathrm{T}$ cell selection and autoreactivity due to a specific genetic defect cause spontaneous autoimmunity that targets the joints.

\section{Table 1}

Effect of cytokine blockade on clinical arthritis in animal models and in RA.

$\begin{array}{lccccccc}\text { Model } & \text { T cell dependence } & \text { TNF- } \alpha & \text { IL-1 } & \text { IL-6 } & \text { IL-4 } & \text { IL-10 } & \text { IFN- } \gamma \\ \text { SKG } & \text { Yes } & \downarrow \downarrow \downarrow & \downarrow \downarrow & \downarrow \downarrow \downarrow & 0 & \uparrow & 0 \\ \text { Active CIA } & \text { Yes then No } & \downarrow \downarrow & \downarrow \downarrow & \downarrow \downarrow \downarrow & \uparrow & \uparrow & \uparrow \uparrow \\ \text { Passive CIA } & \text { No } & \downarrow \downarrow \downarrow & \downarrow \downarrow \downarrow & 0 & & \downarrow & \\ \text { K/BxN } & \text { Yes then No } & 0 & \downarrow \downarrow \downarrow & & & & \\ \text { Passive K/BxN } & \text { No } & \downarrow & \downarrow \downarrow \downarrow & 0 & & & \\ \text { SCW arthritis } & \text { No then Yes } & \downarrow & \downarrow & & \uparrow & \uparrow & \uparrow \\ & & & & & & & \\ \text { Rheumatoid Arthritis } & ? & \downarrow \downarrow & \downarrow & \downarrow \downarrow & & 0 \text { A } & 0^{\text {A }}\end{array}$

$\downarrow$, cytokine blockade suppresses arthritis; $\uparrow$, cytokine blockade increases arthritis; 0 , minimal or no role; AAdministration of exogenous cytokine is ineffective; Badministration of exogenous IFN- $\gamma$ suppresses disease. Data derived from refs. 8-11 and refs. 18-28. SCW, streptococcal cell wall. 
Identification of a similar single mutation in RA is unlikely; it is a multigenic disease where specific sequences in the class II MHC gene have the greatest genetic influence, and identification of other genes remains the subject of intense investigation. Polymorphisms in several cytokine promoters have been implicated, and many genes that regulate $\mathrm{T}$ cell reactivity might also contribute. Nevertheless, the notion that abnormalities in $\mathrm{T}$ cell selection or reactivity on a genetic basis (even if it involves several genes) contribute to synovial inflammation is a plausible explanation for the initiation of RA.

\section{Cytokine networks as a final common pathway}

Several interesting questions with therapeutic importance are raised by these studies. In the early 1990s, the observation that macrophage and fibroblast factors dominate the rheumatoid joint contributed to the hypothesis that cytokine networks, perhaps autonomously, perpetuate RA $(6,7)$. The efficacy of cytokine inhibitors (e.g., TNF- $\alpha$, IL-1, and most recently IL-6) in established and early RA supported this hypothesis (8-10). Synovitis flares after cessation of anticytokine therapy, which clearly indicates that cytokine networks alone are not responsible for chronic disease.

Hence the intriguing nature of the SKG model: even though it is clearly $\mathrm{T}$ cell-dependent (at least for initiation), key macrophage-derived cytokines implicated in RA, such as TNF- $\alpha$, are required for full expression of disease. Sufficient data have now accumulated in other models to suggest that blockade of the inflammatory macrophage-fibroblast axis can be effective regardless of the proximal events that trigger the disease. Table 1 shows that this is true in many other models, including passive and active collagen-induced arthritis (CIA), the native $\mathrm{K} / \mathrm{BxN}$ model, passive serum transfer from $\mathrm{K} / \mathrm{BxN}$ mice, and streptococcal cell wall arthritis. The etiology of these models varies from immunization with auto- or xeno-antigens, injection with antibodies that fix complement in the joint, or activation of innate immunity through toll-like receptors followed by adaptive immune responses. The proinflammatory cytokine network can serve as a final common pathway regardless of the initiating event. There are subtle differences between the models and specific cytokines, such as the widely variable role of IL-6, reinforcing the beliefs that (a) these animal models are not exactly the same as RA in humans, in which the efficacy of TNF- $\alpha$ inhibitors is not nearly as complete as it is in mice; and (b) specific initiating events can have some distinctive cytokine characteristics.

\section{T cell contribution to synovitis}

The role of $\mathrm{T}$ cell cytokines has also been studied in murine systems (see Table 1). Th2 cytokines, like IL-4 and IL-10, generally play an inhibitory role in models mediated by Th1 cells. Of interest, only the latter appears relevant in the SKG model while the prototypic Th 1 cytokine IFN- $\gamma$ contributes very little. In contrast, IFN- $\gamma$ is a suppressive cytokine in many other animal models. The experience with $\mathrm{T}$ cell cytokine modulation in RA is less robust; administration of IL-10 offers little or no benefit (11). Although IFN- $\gamma$ administration does not cause the flares in RA that might be anticipated in a putatively Th1-mediated disease, it also does not have a significant anti-inflammatory effect (12).

Traditional anticytokine interventions can, therefore, be effective in synovial inflammatory diseases regardless of the dependence of arthritis on $\mathrm{T}$ cells. The possibility that subtle abnormalities in $\mathrm{T}$ cell selection and function might initiate or perpetuate RA suggests the utility of alternative approaches that modulate the immune rheostat. Merely killing T cells is not enough to treat RA, as experience with anti-CD4,-CD5, and -CD52 antibody therapeutic interventions have clearly demonstrated (13). Adjusting immune responses by modifying costimulation represents one mechanism that might be useful not only in animal models like SKG but also in humans. A recent clinical study using CTLA4Ig, which blocks the interaction of CD80/86 on dendritic cells with CD28 on naive $\mathrm{T}$ cells, to treat RA is very promising (14). However, like anticytokine therapy, clear efficacy appears to be limited to a subset of patients. Widely variable responses to virtually any treatment modality in RA (besides corticosteroids) suggest that the disease is quite heterogeneous. Unlike treatment for inbred mice with a single gene defect or a single inciting antigen, therapeutic interventions in humans might require individualized medicine that takes into account the genotype and etiologic agent of each patient.

Another lesson learned from this and other animal models is that synovitis can result either from a broad array of immune responses, including those directed against a specific joint antigen (type II collagen), a systemic antigen that can fortuitously localize to the joint (glucose 6-phosphoisomerase), or from generalized $\mathrm{T}$ cell hyperreactivity with multiple autoantigens (ZAP-70 mutations). The latter model is especially intriguing because it suggests that the search for a single pathogenic "rheumatoid antigen" might be fruitless. There is no need to assume that the autoreactive T cells that escape selection in RA must be universally directed towards a unique epitope, and many pathways might lead to this disease. Despite years of research and the tantalizing class II MHC associations, no specific antigen has been found (15). A pattern of autoreactivity occurs instead, which in some cases antedates clinical disease by many years, as demonstrated by the production of anti-CCP antibodies and rheumatoid factors (16). The contribution of T cells in the joints can also be related to antigenindependent mechanisms, such as direct cell contact, which can induce cytokine and protease production by macrophages and fibroblasts (17).

The struggles of Sisyphus were ultimately futile, but scientific progress in understanding RA and autoimmunity advances incrementally with each discovery. One does need to be wary of concluding that the problems underlying the development of RA have been solved with a mouse model. Development of the SKG mouse, however, is one more important step on this pathway of discovery and has provided unique insights into how defective $T$ cell selection can produce inflammatory arthritis and autoreactivity. One key to understanding the utility of the model will be the ability to translate the discovery into novel therapeutic interventions that can be tested in our patients.

Address correspondence to: Gary S. Firestein, Division of Rheumatology, Allergy and Immunology, University of California San Diego School of Medicine, 9500 Gilman Drive, La Jolla, California 92093-0656, USA. Phone: (858) 822-0480; Fax: (858) 534-2359; E-mail: gfirestein@ucsd.edu.

\footnotetext{
1. Hata, H., et al. 2004. Distinct contribution of IL-6, TNF- $\alpha$, IL- 1 , and IL-10 to T cell-mediated spontaneous autoimmune arthritis in mice. J. Clin. Invest. 114:582-588. doi:10.1172/JCI200421795.

2. Pribila, J.T., and Shimizu, Y. 2003. Signal transduction events regulating integrin function and $T$ cell migration: new functions and complexity. Immunol. Res. 27:107-128.

3. Sakaguchi, N., et al. 2003. Altered thymic T-cell selection due to a mutation of the ZAP-70 gene causes autoimmune arthritis in mice. Nature.
} 
426:454-460.

4. Korganow, A.S., et al. 1999. From systemic T cell self-reactivity to organ-specific autoimmune disease via immunoglobulins. Immunity. 4:451-61.

5. Gringhuis, S.I., et al. 2000. Displacement of linker for activation of $\mathrm{T}$ cells from the plasma membrane due to redox balance alterations results in hyporesponsiveness of synovial fluid $\mathrm{T}$ lymphocytes in rheumatoid arthritis. J. Immunol. 164:2170-2179.

6. Firestein, G.S., and Zvaifler, N.J. 1990. How important are $\mathrm{T}$ cells in chronic rheumatoid synovitis? Arthritis Rheum. 33:768-773.

7. Firestein, G.S., and Zvaifler, N.J. 2002. How important are $T$ cells in chronic rheumatoid synovitis? II. T cell-independent mechanisms from beginning to end. Arthritis Rheum. 46:298-308.

8. Nishimoto, N., et al. 2004. Treatment of rheumatoid arthritis with humanized anti-interleukin-6 receptor antibody: a multicenter, double-blind, placebo-controlled trial. Arthritis Rheum. 50:1761-1769.

9. Jiang, Y., et al. 2000. A multicenter, double-blind, dose-ranging, randomized, placebo-controlled study of recombinant human interleukin-1 receptor antagonist in patients with rheumatoid arthritis: radiologic progression and correlation of Genant and Larsen scores. Arthritis Rheum. 43:1001-1009.

10. Moreland, L.W., et al. 1999. Etanercept therapy in rheumatoid arthritis. A randomized, controlled trial. Ann. Intern. Med. 130:478-486.

11. Smeets, T.J., Kraan, M.C., Versendaal, J., Breedveld, F.C., and Tak, P.P. 1999. Analysis of serial synovial biopsies in patients with rheumatoid arthritis: description of a control group without clinical improvement after treatment with interleukin 10 or placebo. J. Rheumatol. 26:2089-2093.
12. Cannon, G.W., et al. 1989. Double-blind trial of recombinant gamma-interferon versus placebo in the treatment of rheumatoid arthritis. Arthritis Rheum. 32:964-973.

13. Moreland, L.W., Heck, L.W., Jr., and Koopman, W.J. 1997. Biologic agents for treating rheumatoid arthritis. Concepts and progress. Arthritis Rheum. 40:397-409.

14. Kremer, J.M., et al. 2003. Treatment of rheumatoid arthritis by selective inhibition of T-cell activation with fusion protein CTLA4Ig. N. Engl. J. Med. 349:1907-1915.

15. Buckner, J.H., and Nepom, G.T. 2002. Genetics of rheumatoid arthritis: is there a scientific explanation for the human leukocyte antigen association? Curr. Opin. Rheumatol. 14:254-259.

16. Nielen, M.M., et al. 2004. Specific autoantibodies precede the symptoms of rheumatoid arthritis: a study of serial measurements in blood donors. Arthritis Rheum. 50:380-386.

17. Burger, D., and Dayer, J.M. 2002. The role of human T-lymphocyte-monocyte contact in inflammation and tissue destruction. Arthritis Res. 3(Suppl):S169-S176.

18. Johansson, A.C., Hansson, A.S., Nandakumar, K.S., Backlund, J., and Holmdahl, R. 2001. IL-10-deficient B10.Q mice develop more severe collageninduced arthritis, but are protected from arthritis induced with anti-type II collagen antibodies. J. Immunol. 167:3505-3512.

19. Alonzi, T., et al. 1998. Interleukin 6 is required for the development of collagen-induced arthritis. J. Exp. Med. 187:461-468.

20. Joosten, L.A., et al. 1997. Role of interleukin-4 and interleukin-10 in murine collagen-induced arthritis. Protective effect of interleukin-4 and interleukin-10 treatment on cartilage destruction.
Arthritis Rheum. 40:249-260.

21. Joosten, L.A., et al. 1999. IL-1 $\alpha \beta$ blockade prevents cartilage and bone destruction in murine type II collagen-induced arthritis, whereas TNF- $\alpha$ blockade only ameliorates joint inflammation. J. Immunol. 163:5049-5055.

22. Guedez, Y.B., et al. 2001. Genetic ablation of interferon-gamma up-regulates interleukin-1beta expression and enables the elicitation of collageninduced arthritis in a nonsusceptible mouse strain. Arthritis Rheum. 44:2413-2424.

23. Kagari, T., Doi, H., and Shimozato, T. 2002. The importance of IL-1 beta and TNF-alpha, and the noninvolvement of IL- 6 , in the development of monoclonal antibody-induced arthritis. J. Immunol. 169:1459-1466.

24. Ji, H., et al. 2002. Critical roles for interleukin 1 and tumor necrosis factor alpha in antibody-induced arthritis. J. Exp. Med. 196:77-85.

25. Kyburz, D., Carson, D.A., and Corr, M. 2000. The role of CD40 ligand and tumor necrosis factor alpha signaling in the transgenic $\mathrm{K} / \mathrm{BxN}$ mouse model of rheumatoid arthritis. Arthritis Rheum. 43:2571-2577.

26. Kuiper, S., et al. 1998. Different roles of tumour necrosis factor alpha and interleukin 1 in murine streptococcal cell wall arthritis. Cytokine. 10:690-702.

27. Lubberts, E., Joosten, L.A., Helsen, M.M., and van den Berg, W.B. 1998. Regulatory role of interleukin 10 in joint inflammation and cartilage destruction in murine streptococcal cell wall (SCW) arthritis. More therapeutic benefit with IL-4/IL-10 combination therapy than with IL-10 treatment alone. Cytokine. 10:361-369.

28. Allen, J.B., et al. 1991. Suppression of bacterial cell wall-induced polyarthritis by recombinant gamma interferon. Cytokine. 3:98-106. 